\title{
E-BOT PLATAFORMA ROBÓTICA DE APOYO EN LA ENSEÑANZA DE ALGORITMOS Y PROGRAMACIÓN
}

\author{
E-bot robotic support platform for teaching algorithms and programming ${ }^{1}$
}

JEFFERSON DAVID GUTIÉRREZ BERNAL ${ }^{2}$, NICOLÁS RICARDO CALDERÓN GONZÁLES ${ }^{3}$ JOSÉ ALEJANDRO FRANCO CALDERÓN ${ }^{4}$

Recibido:20 de abril de 2021. Aceptado:20 de junio de 2021

DOI: http://dx.doi.org/10.21017/rimci.2021.v8.n16.a103

\begin{abstract}
Resumen
Este artículo presenta el diseño y la construcción de un robot móvil (E-BOT) tanto en la parte de hardware como en la de software, el cual funciona como una herramienta de apoyo a los procesos de enseñanza y permite afianzar el aprendizaje en temas relacionados a programación teniendo como alcance las estructuras repetitivas, esto por medio de una serie de elementos de aprendizaje previamente construidos.

E-BOT cuenta con una serie de actuadores (Led, oled, servomotor, motores) y sensores (ultrasonido y luz reflectante) los cuales son controlados por medio de Arduino que permiten una fácil interacción de EBOT con el mundo real. Adicionalmente se realiza la construcción de un software de verificación que nos permite validar el correcto funcionamiento de todos los módulos construidos.
\end{abstract}

Palabras Clave. Robotica educativa; Robotica Movil; diseño y construcción; Software de verificación; Sensores; y Actuadores.

\begin{abstract}
This article presents the design and construction of a mobile robot (E-BOT) both in the hardware and in the software part, which works as a support tool for teaching processes and allows to consolidate learning in related topics. a Programming having as its scope repetitive structures, this through a series of previously constructed learning elements.

E-BOT has a series of actuators (Led, oled, servomotor, motors) and sensors (ultrasound and reflective light) which are controlled by means of Arduino that allow an easy interaction of EBOT with the real world. Additionally, the construction of a verification software is carried out that allows us to validate the correct operation of all the modules built.

Keywords. Educational robotics; Mobile Robotics; design and construction; Verification software; Sensors; and Actuators.
\end{abstract}

\section{INTRODUCCIÓN}

L XXI plantean la necesidad de redefinir los modelos de enseñanza y aprendizaje para potenciar en los estudiantes el desarrollo de capacidades relacionadas con la creatividad y la resolución de problemas, unidas a la adquisición

1 Trabajo de semillero de investigación del grupo GIDIS de la Corporación Universitaria Republicana

2 Ingeniero de sistemas. Corporación Universitaria Republicana. Correo electrónico: jefferson.gutierrez2030@gmail.com

3 Ingeniero de sistemas. Corporación Universitaria Republicana. Correo electrónico: nico.calderon04@gmail.com

4 Ingeniero Electrónico y Magister en electrónica de la Escuela Colombiana de Ingeniería "Julio Garavito", especialista en diseño de aplicaciones para televisión digital interactiva y en administración de tecnologías de la información para la comunicación virtual de la Universidad Manuela Beltrán, Docente investigador de la facultad de ingeniería adscrito al Grupo de Investigación y Desarrollo en Ingeniería de Sistemas - GIDIS de la Corporación Universitaria Republicana. Correo electrónico: alejing@urepublicana.edu.co. ORCID: https://orcid.org/0000. $0003-3931-2186$ 
de competencias técnico-científicas que permitan resolver los retos inciertos del futuro [1]. Por esta razón la robótica en los últimos años se ha convertido en una disciplina, muy popular y con gran implementación en diferentes ámbitos como medicina, seguridad, automatización, entre otras. Sin embargo, es en la educación donde ha generado un gran impacto, dado que puede ser utilizada para enseñar otras asignaturas de una manera más dinámica [2].

Es así como en [3] resaltan la importancia de la robótica en el ámbito educativo y se convierte en un recurso para facilitar el aprendizaje que permite desarrollar competencias generales como la socialización, la creatividad y la iniciativa, que ayude al estudiante a dar una respuesta adecuada a los entornos cambiantes del mundo actual. La presencia de la robótica en el aula de clase no intenta formar a los estudiantes en la disciplina de la robótica propiamente dicha, sino aprovechar su carácter multidisciplinar para generar ambientes de aprendizaje donde el estudiante pueda percibir los problemas del mundo real, imaginar y formular las posibles soluciones y poner en marcha sus ideas, mientras se siente motivado por los temas que se van desarrollando.

En [4][5] afirman que algunos estudios indican que la educación mediante la interacción con los robots añade posibilidades al enfoque tradicional centrado en la construcción y programación de estos ultimos. La principal suposición de este enfoque es que la interacción con los robots puede reforzar los procesos educativos y los resultados, tales como el aprendizaje conceptual y el entrenamiento cognitivo, motivar a los estudiantes, apoyar la curiosidad y aumentar la conciencia sobre la interacción entre otras ciencias aplicadas y la robótica.

\section{Problema}

El presente trabajo nace de la necesidad de estudiar como la robótica educativa puede mejorar los procesos de enseñanza y aprendizaje en la programación por medio de plataformas roboticas construidas y diseñadas para este fin creando rutas que permitan abordar el conocimiento como un todo, permitiendo enseñar de una manera lúdica promoviendo así un aprendizaje significativo.

\section{ESTADO DEL ARTE}

En [3] Se muestra la importancia que tiene el uso de la robótica como una herramienta de aprendizaje y presenta las etapas típicas que se deben afrontar al implementar proyectos de robótica educativa en el aula de clase. También se da a conocer un proyecto de robótica educativa denominado "Mundo Robótica" el cual busca involucrar robots en el aula de clase por medio de actividades prácticas y recursos de aprendizaje articulados desde una plataforma virtual. Al final del proyecto se concluye que la implementación de proyectos de robótica educativa en el aula de clase crea mejores condiciones de apropiación de conocimiento, las cuales permiten a los estudiantes fabricar sus propias representaciones de los fenómenos del mundo que los rodea, facilitando la adquisición de conocimientos acerca de estos fenómenos y su transferencia a diferentes áreas del conocimiento.

En [6] se presenta la experiencia en la utilización de un robot móvil "Piero", para motivar a los estudiantes en una asignatura de grado de ingeniería, que combina las metodologías activas de docencia tales como, la clase invertida, el aprendizaje basado en proyectos, y el aprendizaje colaborativo; junto con el empleo de herramientas TIC como el portafolio electrónico y vídeo tutoriales. Al final del estudio se concluyó que el uso del robot aumentó en alto grado la motivación de los estudiantes sin embargo se evidencia que el principal pendiente de la investigación es la documentación del aprendizaje autónomo, la elaboración de una guía para la creación de contenidos normalizados en el portafolio digital. Ya que todo el proceso de enseñanza debía estar bajo la supervisión docente.

En [7] se diseñó e implementó una plataforma integrada por un sistema de gestión de aprendizaje configurado en una tarjeta Raspberry $\mathrm{Pi}$, un módulo de adquisición de datos conformado por una tarjeta Arduino y un elemento de interacción con el usuario representado en un dispositivo móvil. El sistema fue concebido como una herramienta para la enseñanza de las ciencias naturales y se implementó con un estudio de caso en el área Física. El uso de un sistema integrado con dispositivos tecnológicos motivó el interés por el aprendizaje de los conceptos relacionados con el funcionamiento de estos. Lo anterior se infiere a partir de que

Rev. Ingeniería, Matemáticas y Ciencias de la Información Vol. 8 / Núm. 16 / julio - diciembre de 2021; 65-76 
los estudiantes manifestaran una mejor disposición para el desarrollo de temáticas asistidas con ayuda de las TIC. Igualmente, la posibilidad de interactuar con sus compañeros y con los montajes experimentales propios del área de estudio, generaron un ambiente propicio para el aprendizaje activo.

En [8] se realiza la investigación del impacto de distintas tecnologías y metodologías de enseñanza utilizadas y que tienen el potencial de mejorar el aprendizaje de distintos temas de las Ciencias Informáticas tomando como base la gamificación sin embargo, a lo largo de la investigación se encuentran otros estilos de enseñanza entre ellos la robótica educativa en el que afirma que "los robots cuentan con el potencial de facilitar el aprendizaje de un lenguaje de programación, propiciar la experimentación y estimular las competencias asociadas a la resolución de problemas, permite concretizar la relación existente entre software y hardware, y hacen posible además que el estudiante obtenga un resultado palpable de su producción, aportando entonces a la idea de "construccionismo", que afirma que el conocimiento se construye".

En [9] se realiza una investigación experimental, la población está constituida por 179 estudiantes del nivel secundario de la I.E. "General José de San Martín" de la Provincia Arequipa y se trabajó con una muestra no probabilística de tipo intencional de 33 estudiantes, para la recolección de datos se utilizaron rúbricas de evaluación, aplicadas en un Pre Test al inicio del curso y un Post Test al final del curso; para lograr lo resultados se elaboraron 8 sesiones de aprendizaje del taller de robótica básica, diseñadas con un enfoque por competencias y una orientación al aprendizaje colaborativo, como resultado se acepta la hipótesis planteada: La robótica educativa influencia el aprendizaje colaborativo de los estudiantes de primero de secundaria de la IE general José de San Martín.

En [10] se desarrolló una investigación la cual permitió reconocer la importancia de la articulación entre la robótica educativa con el desarrollo de las habilidades de pensamiento y la creatividad. Por medio de dispositivos Lego. Se Concluye que el uso de materiales y de soportes didácticos como Lego apoya el desarrollo de la clase de tec- nología lo cual permite que el interés y hace que la creatividad sea mayor por parte de los estudiantes analizados.

En [11] se plantea cómo el uso de la robótica puede ampliar de manera significativa la gama de actividades que pueden ser desarrolladas y promover la integración entre diferentes áreas del conocimiento, debido a su gran potencial como herramienta de uso multidisciplinar, a través de la práctica científica, la simulación de mecanismos o la construcción de prototipos (en este caso se realiza la construcción de Edubot v-2) mediante la metodología "learning-by-doing".

En [12] se analiza la robótica educativa como una forma de trabajo que sustenta el desarrollo del pensamiento computacional en niños, niñas y jóvenes de educación general, más allá de la programación, y de las orientaciones profesionales a las que se dediquen en el futuro. Se presenta la programación como una fase del trabajo en proyectos específicos elegidos por los estudiantes.

En [13] se realiza un estudio pretende averiguar si la utilización del robot "Bee-Bot" puede mejorar el aprendizaje y favorecer el interés del alumnado por las ciencias naturales. Para resolver esta cuestión, se realiza una intervención didáctica basada en el uso del robot y se compara con una metodología más tradicional basada en la exposición de los contenidos sin la utilización del robot educativo. Concluyendo su estudio en que el uso de la robótica en el currículo escolar permite que los contenidos curriculares se enseñen de manera práctica, despertando curiosidad y promoviendo una metodología científica de construcción de conocimiento.

En [14] se desarrolla un planteamiento transversal, en el que el robot se convierte en una herramienta activa para que los estudiantes aprendan contenidos propios del currículo oficial de diferentes disciplinas, a la vez que desarrollan otro tipo de competencias transversales, como el pensamiento computacional. Los resultados muestran que el uso de robots como herramienta de apoyo en contextos educativos con alumnos de edades tempranas (7-8 años) aporta beneficios en diferentes dimensiones del proceso de enseñanza-aprendizaje. Se perciben mejoras en la adquisición de contenidos curriculares transversales 
relacionados con la orientación espacial e interpretación de planos.

En [15] se realiza la construcción de la plataforma "StrandBotic" la cual es un robot de bajo costo utilizando hardware y software libre para que estudiantes aprendan a pensar algorítmicamente. En este contexto, el robot sólo es una excusa para trabajar conceptos de pensamiento computacional y despertar vocaciones científicas. Este trabajo desarrolla y pone a disposición de la comunidad educativa una plataforma completa, accesible y documentada que puede ser utilizada como un recurso más dentro del aula para la enseñanza de la programación en las escuelas de nivel primario.

\section{MARCO TEÓRICO}

\section{A. Robótica pedagógica}

A través de la integración de diferentes áreas del conocimiento, es posible la obtención de considerables resultados. La robótica es un ejemplo de la integración de diferentes áreas del conocimiento; a través de esta disciplina se integran sistemas mecánicos, eléctricos, electrónicos, informáticos y de comunicaciones.

La "Robótica Pedagógica" en [16] es definida como una disciplina que permite concebir, diseñar y desarrollar robots educativos para que los estudiantes se inicien desde muy jóvenes en el estudio de las ciencias y la tecnología; surge con la finalidad de explotar el deseo de los educandos por interactuar con un robot para favorecer los procesos cognitivos. Vivet y Nonnon, en 1989 definen esta disciplina como: "La actividad de concepción, creación y puesta en funcionamiento, con fines didácticos, de objetos tecnológicos, que son reproducciones reducidas muy fieles y significativas de los procesos y herramientas robóticas que son usadas cotidianamente, sobre todo, y que cada vez son más comunes en nuestro entorno social, productivo y cultural".

Una característica especial que tiene la robótica educativa es la capacidad de mantener la atención del estudiante. El hecho de que el estudiante pueda manipular y experimentar con estas herramientas de aprendizaje basadas en maquinas hace que pueda centrar sus percepciones y observaciones en la actividad que está realizando. Un testimonio en este sentido lo dan Pierre Nonnon y Jean Pierre Theil, quienes afirman que el uso de herramientas robóticas favorece el proceso de enseñanza-aprendizaje, pues permite fácilmente la integración de lo teórico con lo práctico, el desarrollo de un pensamiento sistémico y la adquisición de nociones científica [3].

\section{B. Microcontrolador}

Un microcontrolador es un dispositivo electrónico capaz de llevar a cabo procesos lógicos. Estos procesos o acciones son programados en lenguaje ensamblador por el usuario, y son introducidos en este a través de un programador [17].

\section{Arduino}

En [18] con el fin de entender que es un Arduino se hace relación la compresión desde 3 frentes, estos son:

1. Una placa de hardware libre que incorpora un microcontrolador reprogramable y una serie de pines que permite la conexión de sensores y actuadores.

2. Un software (mas en concreto un entorno de desarrollo libre y multiplataforma que debemos instalar en el ordenador que permite escribir verificar y guardar (o cargar) en la memoria del microcontrolador de la placa de Arduino el conjunto de instrucciones que deseamos que este empiece a ejecutar.

3. Un lenguaje de programación libre por "lenguaje de programación" se entiende cualquier idioma artificial diseñado para expresar instrucciones (siguiendo unas determinadas reglas sintácticas) que pueden ser interpretadas y ejecutadas por las maquinas.

\section{PCB (Printed Circuit Board)}

Es una placa de circuito impreso, son superficies fabricadas de un material no conductor (normalmente resinas de fibra de vidrio reforzada cerámica o plástico) sobre las cuales aparecen laminas pegadas junto con pistas de material conductor (normalmente cobre). Las PCB son utilizadas para conectar eléctricamente, a través de caminos conductores diferentes componentes electrónicos 
soldados en ella. La PCB es la forma más compacta y estable de construir un circuito electrónico, pero una vez fabricada su diseño es bastante difícil de modificar [18].

\section{E. Sensor}

Son dispositivos de entrada que recogen información del mundo real y que proveen una salida ya sea analógica o digital, con el fin de que el sistema de control entienda y pueda procesar y ejecutar alguna acción [19].

\section{F. Sensor de línea QTR1RC}

Es un sensor de reflectancia que mide el color de una superficie sólida, su funcionamiento consiste en que un emisor (diodo) envía señales infrarrojas de corto alcance, hacia la superficie permanentemente, el receptor (fototransistor) que se encuentra en paralelo, recibirá ese haz con distinta intensidad dependiendo si la superficie es clara $u$ oscura [20].

\section{G.Sensor ultrasónico HC-SR04}

El HC-SR04 es un sensor de distancias por ultrasonidos capaz de detectar objetos y calcular la distancia a la que se encuentra en un rango de 2 a $450 \mathrm{~cm}$; cuantifica el "delay" de la señal, es decir, el tiempo que demora la señal en regresar el eco [21].

\section{H. Actuadores}

Un actuador es un dispositivo con la capacidad de generar una fuerza que ejerce un cambio de posición velocidad o estado de algún tipo sobre un elemento mecánico a partir de la transformación de energía [21].

\section{Motor CD}

Un motor eléctrico de Corriente Directa (o CD) es una máquina que convierte energía eléctrica en movimiento o trabajo mecánico a través de medios electromagnéticos [22].

\section{J. Servomotor}

Un servomotor (o servo) es un dispositivo similar a un motor de corriente continua que tiene la capacidad de ubicarse en cualquier posición den- tro de su rango de operación y mantenerse estable en dicha posición. Se controla mediante el envío de impulsos eléctricos. Estos pulsos le dicen al servo a que? posición se debe mover [21].

\section{K. Diodo LED}

El diodo LED (Light Emitting Diode) es un diodo que tiene la característica de emitir luz cuando está en polarización directa. La intensidad de luz emitida es proporcional a la intensidad de corriente que circula por el diodo y la longitud de onda depende del material que este fabricado. También existen LEDs que emiten luz con longitudes de onda fuera del rango visible, como los LEDs de luz infrarroja o luz ultravioleta [20].

\section{OLED}

El Organic Light Emitting Diode (OLED) es un diodo emisor de luz formado por una capa muy fina de polímeros orgánicos, es decir, grandes moléculas compuestas de unidades químicas en cadena que son capaces de convertir energía eléctrica en luz cuando están situadas entre dos electrodos [20].

\section{M.Driver L298N}

Es un circuito integrado que permite controlar dos motores de corriente continua o un motor paso a paso bipolar de hasta 2 amperios. El módulo cuenta con todos los componentes necesarios para funcionar sin necesidad de elementos adicionales, entre ellos diodos de protección resistencias y un regulador que suministra 5V a la parte lógica [21].

\section{Propuesta (diseño de E-BOT)}

\section{A. Diseño y contrucción mecánica}

Una vez seleccionado los componentes que integrarían el robot, se procede a realizar el diseño mecanico del mismo. Como base se tomo dos placas de acetato de 19x16 CM. Sobre la primera placa se adecuaron los dos motores DC el controlador L298N, el servomotor y en la parte delantera se ajustaron los tres sensores de linea QTR1RC por medio de una placa de acetato de $3 \times 4 \mathrm{CM}$ y también se añadió una rueda loca como lo muestra la Fig. 1. 


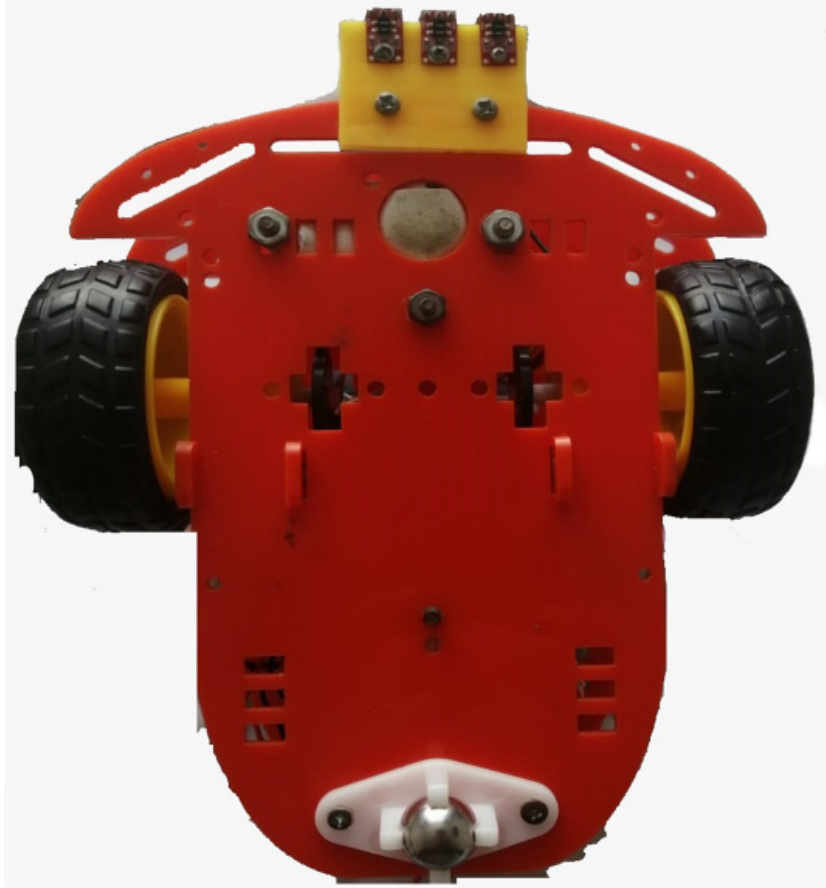

Fig. 1. Vista inferior E-BOT

Sobre la segunda placa y dejando un espacio de 4.5 CM como se puede evidenciar en la Fig. 2, se acopla la segunda placa de acetato, sobre esta se incorpora un molde donde se ajusta el sensor de ultrasonido HC-SR04.

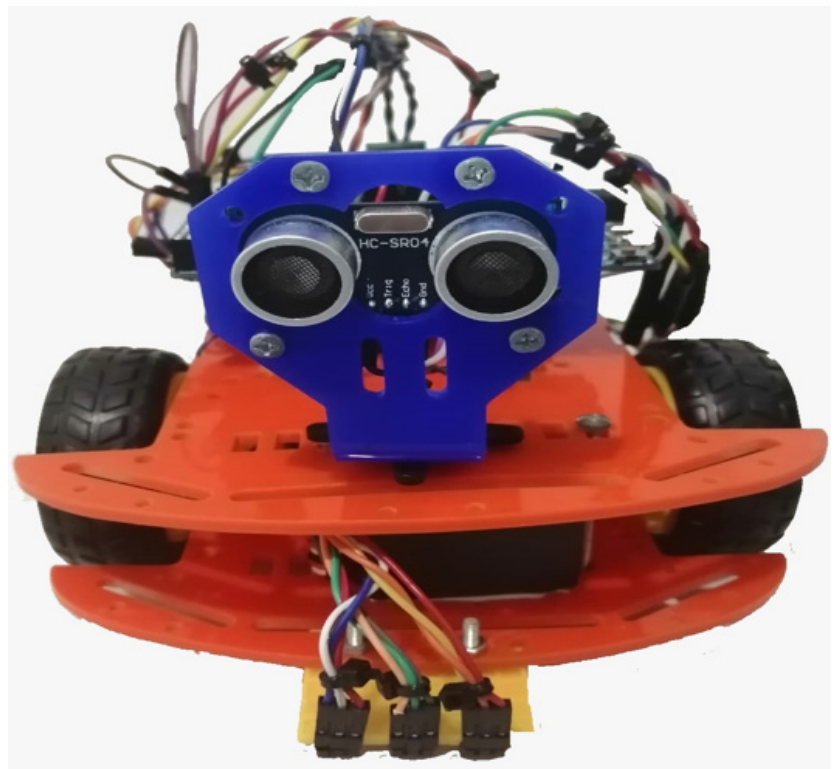

Fig. 2. Vista Frontal E-BOT
Adicionalmente y como se puede observar en la Fig 3, se acopla el Arduino y la placa PCB junto con las conexiones de alimentación, LEDs y OLED.

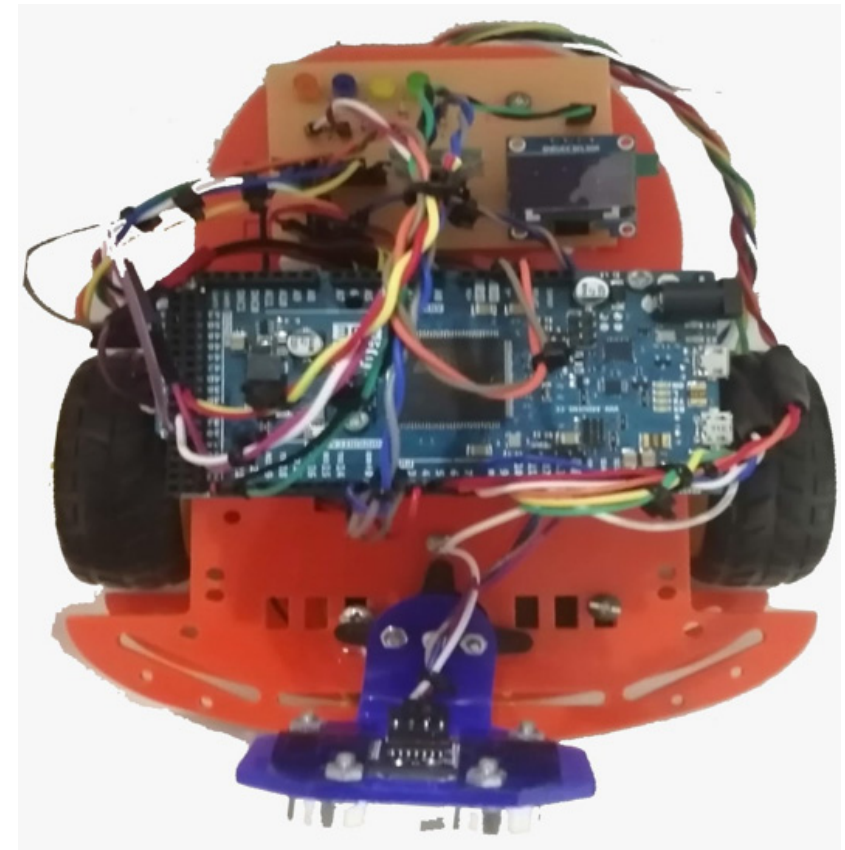

Fig. 3. Vista Superior E-BOT

\section{B. Diseño y contrucción electrónica}

E-BOT se diseñó de forma modular, compuesto por los distintos subsistemas fundamentales, estos se pueden evidenciar a nivel general en la Fig. 4.

\section{- Subsistema de procesamiento de la información}

Basado en el microcontrolador (Arduino DUE) que se encarga de comandar todas las acciones del robot, tales como procesar la información que proviene del puerto de comunicación serial o sensores, para luego enviar órdenes a los motores y demás actuadores.

\section{- Subsistema sensórico}

Está compuesto por un sensor de ultrasonido (HSR04) y 3 sensores de luz reflactante QTR1RC.

\section{- Subsistema actuador}

Se encuentra compuesto por los motores de corriente continua, los LEDs, la pantalla oled y el

Rev. Ingeniería, Matemáticas y Ciencias de la Información Vol. 8 / Núm. 16 / julio - diciembre de 2021; 65-76 


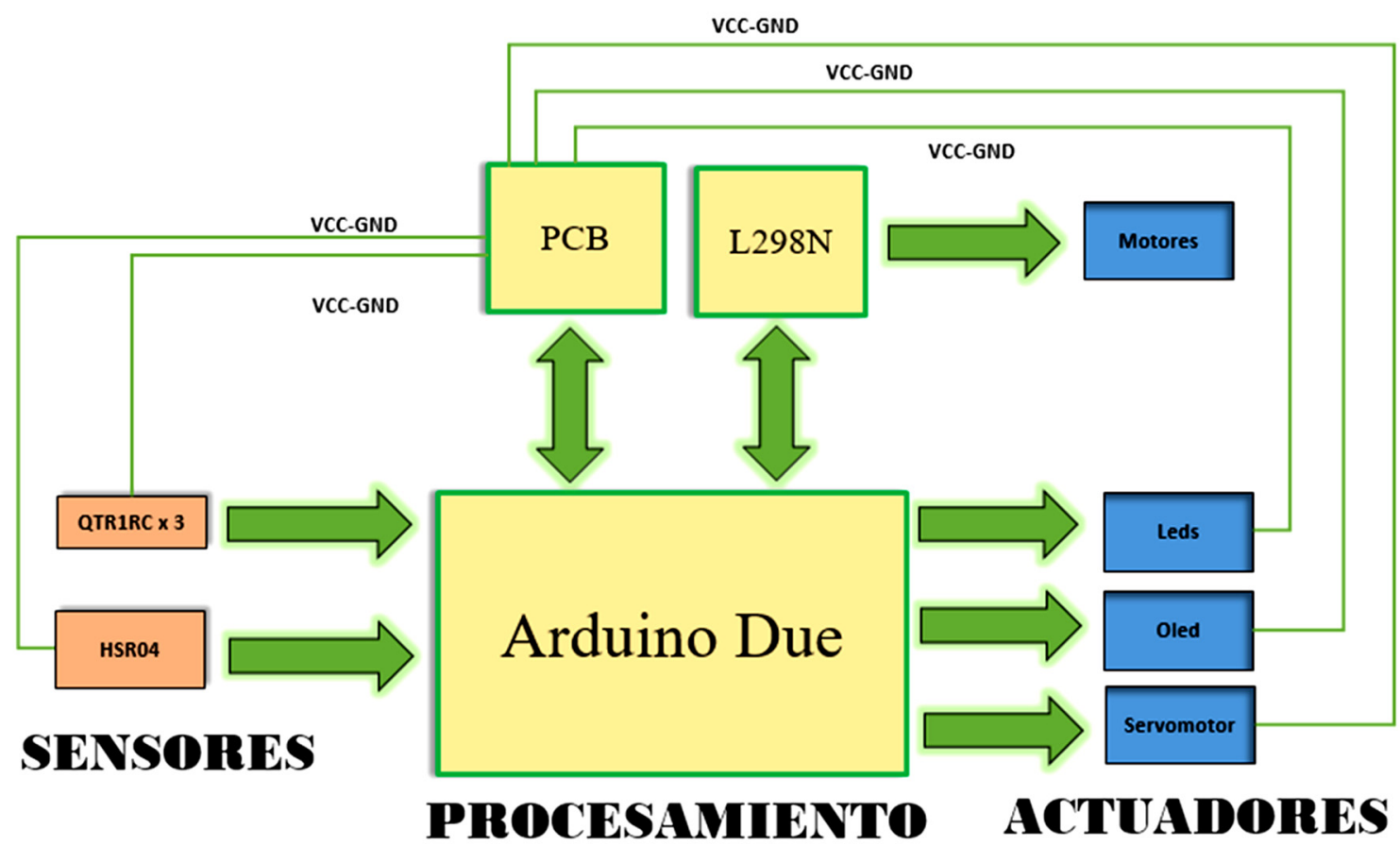

Fig. 4. Diagrama Módular E-BOT

servomotor. Es importante tener en cuenta que tanto los sensores como actuadores se encuentran energizados con ayuda de una placa de circuito impreso (PCB). En la Fig. 5, se muestra el diseño realizado de la misma.
A continuación, en la Tabla I se muestra la distribución de pines y conexiones de los diferentes componentes de E-BOT, cabe resaltar que aun existen algunos pines disponibles en el microcontrolador con el fin de mejorar o aumentar las capacidads del robot.

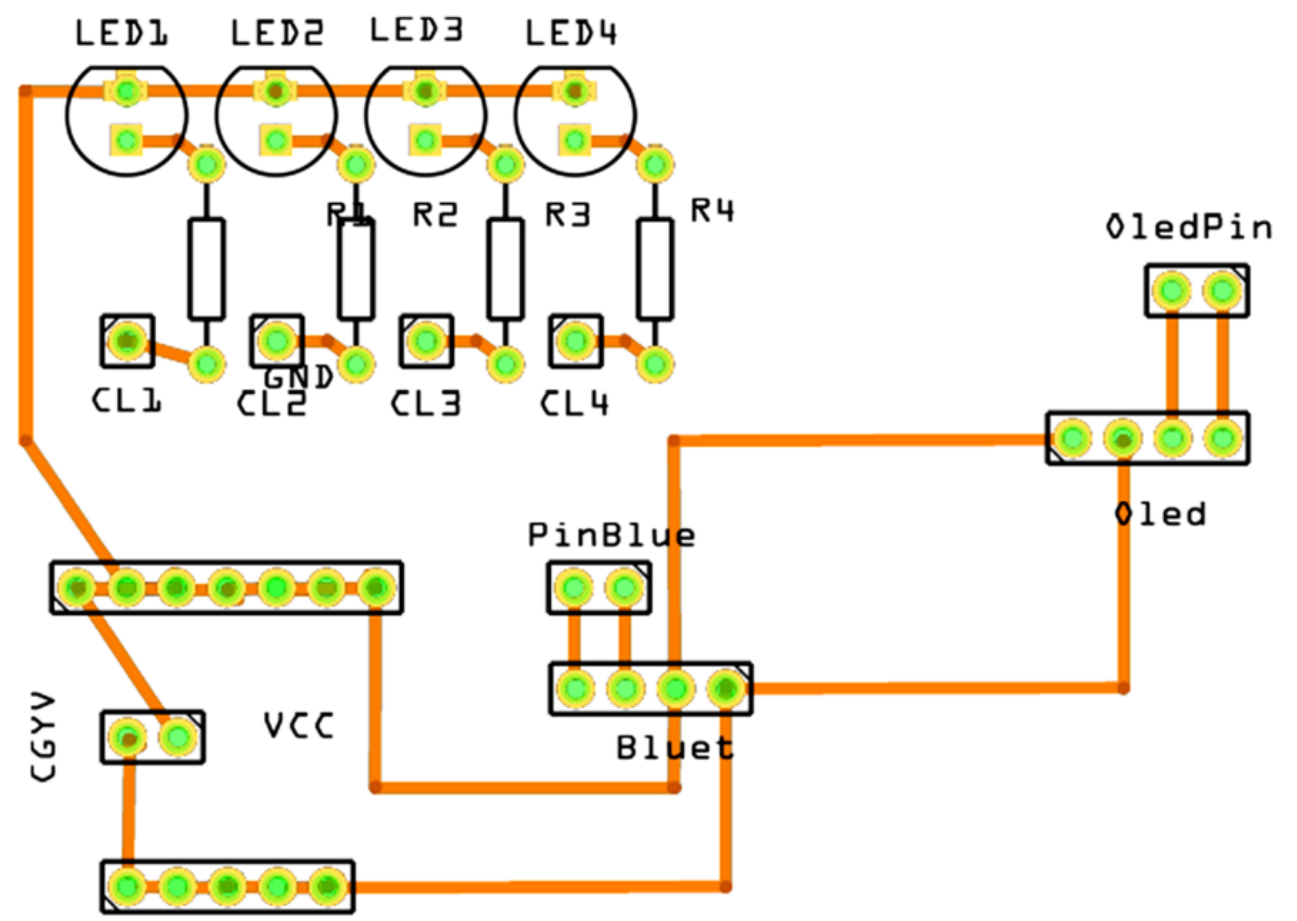

Fig. 5. Diseño de Placa de Circuito Impreso (PCB] 
Tabla I. Conexión de pines

\begin{tabular}{|c|c|c|c|c|}
\hline L298N & ACTUADOR & $\begin{array}{c}\text { ENABLE A } \\
\text { A1 } \\
\text { A2 } \\
\text { ENABLE B } \\
\text { B1 } \\
\text { B2 } \\
\text { VCC } \\
\text { GND }\end{array}$ & $\begin{array}{c}10 \\
9 \\
8 \\
7 \\
6 \\
5 \\
\text { PCB } \\
\text { PCB }\end{array}$ & $\begin{array}{c}\text { DIGITAL PWM } \\
\text { DIGITAL PWM } \\
\text { DIGITAL PWM } \\
\text { DIGITAL PWM } \\
\text { DIGITAL PWM } \\
\text { DIGITAL PWM } \\
\text { VCC } \\
\text { GND }\end{array}$ \\
\hline SERVOMOTOR & ACTUADOR & $\begin{array}{l}\text { PIN DIGITAL } \\
\text { VCC } \\
\text { GND }\end{array}$ & $\begin{array}{c}11 \\
\text { РCB } \\
\text { PCB }\end{array}$ & $\begin{array}{c}\text { DIGITAL PWM } \\
\text { VCC } \\
\text { GND }\end{array}$ \\
\hline OLED & ACTUADOR & $\begin{array}{l}\text { SCL } \\
\text { SDA } \\
\text { VCC } \\
\text { GND }\end{array}$ & $\begin{array}{c}21 \\
20 \\
\text { PCB } \\
\text { PCB }\end{array}$ & $\begin{array}{l}\text { DIGITAL SCL } \\
\text { DIGITAL SDA } \\
\text { VCC } \\
\text { GND }\end{array}$ \\
\hline LEDS & ACTUADOR & $\begin{array}{c}\text { PIN DIGITAL } \\
\text { PIN DIGITAL } \\
\text { PIN DIGITAL } \\
\text { PIN DIGITAL } \\
\text { VCC } \\
\text { GND }\end{array}$ & $\begin{array}{c}30 \\
32 \\
34 \\
36 \\
\text { PCB } \\
\text { PCB }\end{array}$ & $\begin{array}{c}\text { DIGITAL } \\
\text { DIGITAL } \\
\text { DIGITAL } \\
\text { DIGITAL } \\
\text { VCC } \\
\text { GND }\end{array}$ \\
\hline HSR04 & SENSOR & $\begin{array}{l}\text { ECHO } \\
\text { TRIG } \\
\text { VCC } \\
\text { GND }\end{array}$ & $\begin{array}{c}13 \\
12 \\
\text { PCB } \\
\text { PCB }\end{array}$ & $\begin{array}{l}\text { DIGITAL PWM } \\
\text { DIGITAL VCC } \\
\text { GND }\end{array}$ \\
\hline QTR & SENSOR & $\begin{array}{c}\text { PIN DIGITAL } \\
\text { IZQUIERDO PIN DIGITAL. } \\
\text { CENTRO PIN DIGITAL } \\
\text { DERECHO } \\
\text { VCC } \\
\text { GND }\end{array}$ & $\begin{array}{c}5 \\
4 \\
3 \\
\text { PCB } \\
\text { PCB }\end{array}$ & $\begin{array}{c}\text { DIGITAL. PWM } \\
\text { DIGITAL. PWM } \\
\text { DIGITAL PWM } \\
\text { VCC } \\
\text { GND }\end{array}$ \\
\hline
\end{tabular}

Fuente: Los autores.

\section{Diseño y contrucción del software de verifi- cación de funcionamiento para E-BOT}

Una vez construida la parte mecánica y electrónica se observó la necesidad de iniciar con el desarrollo de un software que permitiera evidenciar el funcionamiento de todos los módulos de robot, esto debido a que se requería comprobar que los componentes previamente desarrollados e integrados en E-BOT funcionarán de una manera adecuada y acorde a la necesidad del proyecto.

Se realizó un análisis donde se decidió que el lenguaje de programación elegido sería Python debido a que es un lenguaje que tiene una sintaxis fácil de entender, es adicionalmente sencillo de enseñar (o aprender) y de utilizar en múltiples propósitos y aplicaciones.

Como proceso preliminar se realizó el diseño de la interfaz de verificación como se evidencia en la Fig. 6, en el programa "Balsamiq Mockups" que permitió generar de manera preliminar una interfaz gráfica de usuario.

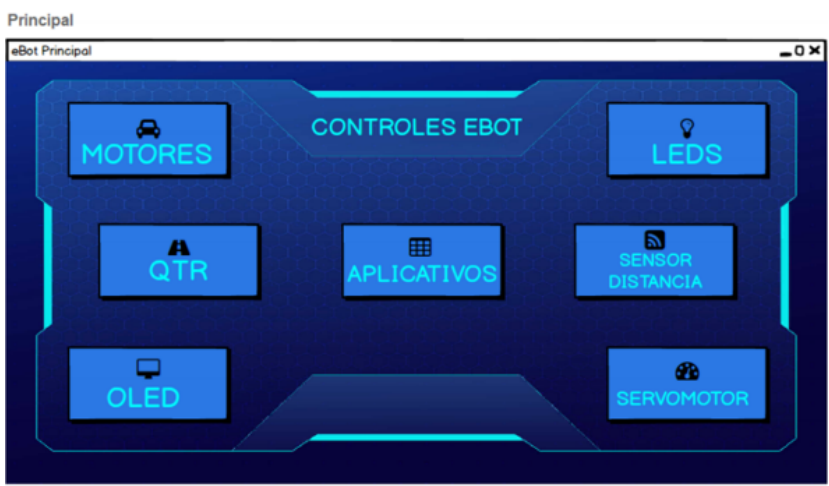

Fig. 6. Mockups Ventana Principal E-BOT

Después de realizar cada una de las ventanas por medio de "Balsamiq Mockups" se realizó la programación de estas. Hay que tener en cuenta que para dicho desarrollo se utilizó el editor de código "Visual Studio Code" y para la parte gráfica fue necesario utilizar "Qt Designer" la cual es una herramienta que nos permite diseñar y construir vistas gráficas hacia el usuario.

Es importante tener presente que todo diseño en "Qt Designer" debe ser programado en Python en "Visual Studio Code" y para realizar esto se debe utilizar la librería "PyQt" la cual es una unión de la biblioteca gráfica de "Qt Designer" con el lenguaje de programación Python.

Como se menciono con anterioridad E-BOT cuenta con 2 sensores y 4 actuadores es por este motivo, para el software de verificación se necesitaba mínimo esta misma cantidad de módulos los cuales serán descritos a continuación.

\section{- Módulo QTR}

Permite medir la reflectancia de una superficie por medio de un conjunto de 3 sensores los cuales cada uno determina si se encuentra sobre una superficie negra o blanca, o de alta o baja reflactancia.

\section{- Módulo de Leds}

Permite el encender y apagar uno o todos los diferentes Leds que se tienen conectados a E-BOT como se muestra en la Fig. 7. 


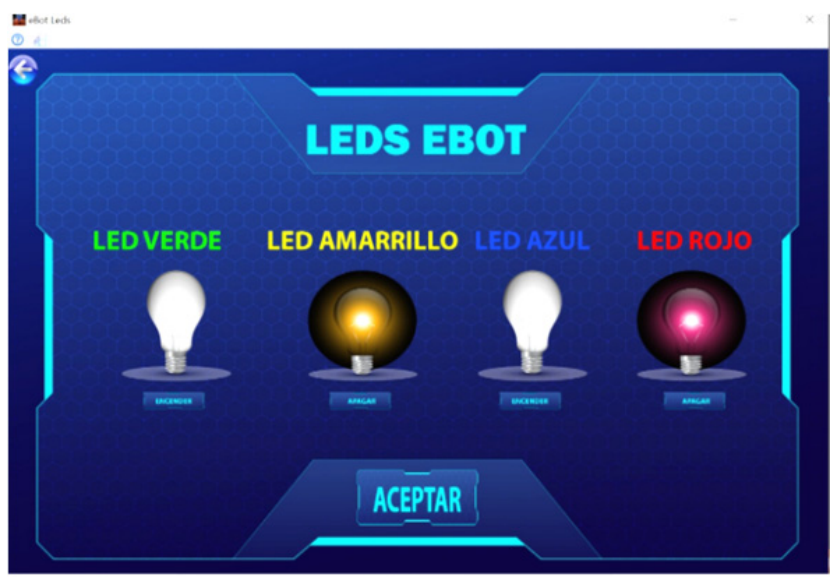

Fig. 7. Módulo Leds E-BOT

\section{- Módulo HC-SR04}

Permite conocer la distancia en centímetros que hay desde E-BOT hasta un objeto.

\section{- Módulo OLED}

Para este módulo se logró configurar 2 funciones las cuales permiten ver el correcto funcionamiento de la pantalla OLED, el primero permite realizar operaciones matemáticas básicas y en el segundo escribir un mensaje, tanto la primera como la segunda función muestran la información en la pantalla OLED dispuesta en E-BOT.

\section{- Módulo de motores}

Permite el desplazamiento de E-BOT según las funciones previamente programadas (adelente, atrás, giro izquierdo, giro derecha y rotación sobre su propio eje) como se muestra en la Fig. 8.

\section{- Módulo servomotor}

Permite activar el servomotor y generar un giro entre $0^{\circ}$ y $180^{\circ}$ según como el usuario quiera configurarlo.

Por último, con el fin de conectar todos módulos de una forma eficiente se han programado dos librerías denomindas "Sensores" y "Actuadores" dentro de sensores se encuentrán las funciones necesarias para interactuar con los sensores QTR y HC-SR04 y dentro de actuadores se encontrarán

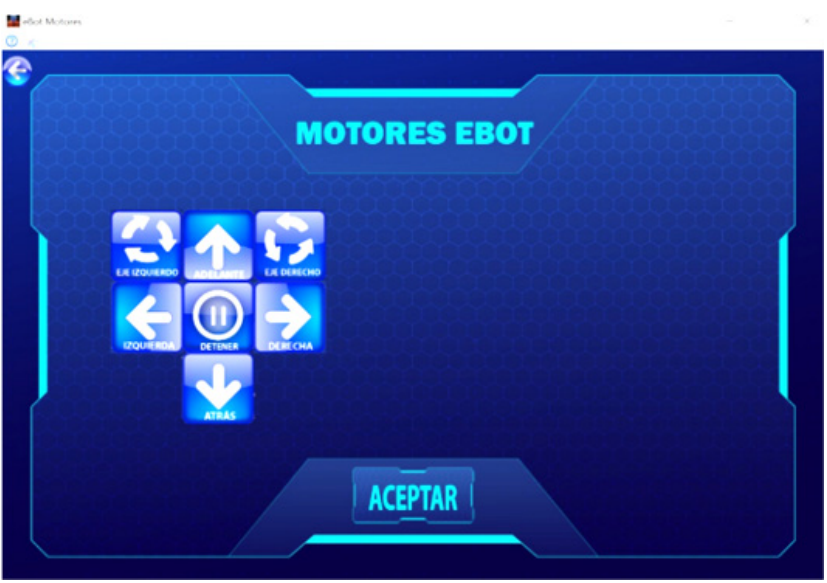

Fig. 8. Módulo Motores E-BOT

el resto de las funciones para interactuar con los módulos explicados anteriormente. Se realizó esto con el fin de diferenciar la parte lógica de la parte gráfica y que el código implementado pueda funcionar en cualquier otra interfaz.

Durante el desarrollo se identificó la necesidad de crear una librería que permitiera la conexión entre Python y Arduino indistintamente del puerto al que este último estuviera conectado, es por lo que se programó una tercera librería "Conexión" la cual desde el inicio establece la comunicación con E-BOT y el computador al solícitar la configuración del puerto, una vez diligenciada esta información esta variable es guardada y se aplica a las demás partes del código. En la Fig. 9, se muestra cuando la variable es solicitada al usuario.

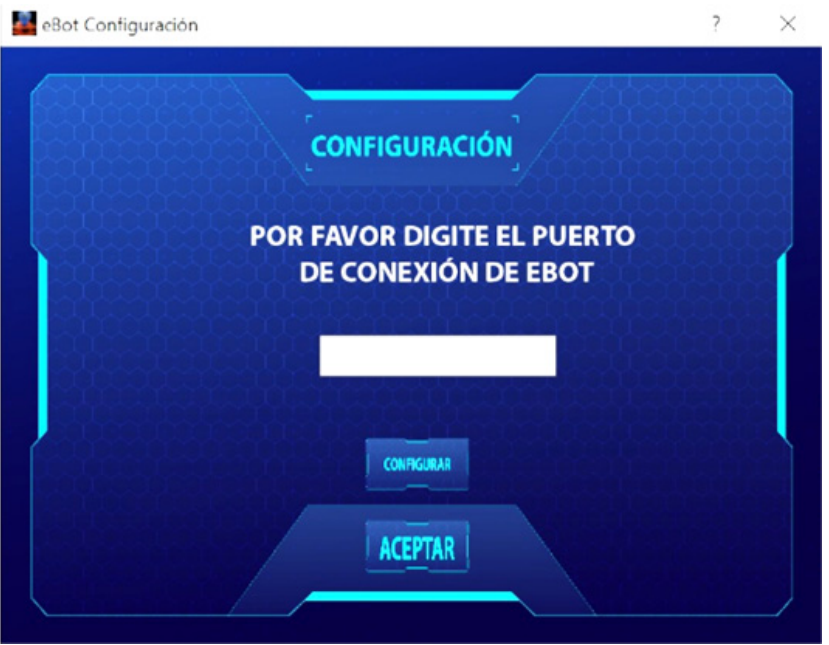

Fig. 9. Configuración de Puerto E-BOT 


\section{Resultados}

Se diseñó un sistema que se caracteriza por su modularidad, su fácil programación y por qué consta de múltiples sensores y actuadores, cuya combinación es ideal para el óptimo desempeño del robot, así mismo se realiza la implementación de un software de verificación el cual permite validar el funcionamiento de todos los subsistemas del robot.

Por otro lado, se han creado 3 propuestas de elementos de aprendizaje, los cuales tienen una serie de actividades que permitirán tener una interacción de E-BOT con el aula de clase, permitiendo un mayor dinamismo en la enseñanza de la programación. Para poder realizar esta integración y como se describe en cada uno de los elementos de aprendizaje es importante realizar la importación de librerías a nuevos proyectos previamente desarrolladas.

A continuación, se realiza una descripción de lo que se podrá enseñar con la aplicación de cada uno de los elementos de aprendizaje y sus actividades propuestas.

\section{- Elemento de aprendizaje 1}

Se realizan actividades relacionadas con los conceptos fundamentales de la programación tales como tipo de datos, operaciones aritméticas y lógicas, a continuación, en la Fig 10, se muestra un ejemplo de una de las actividades contempladas y su integración junto con E-BOT.

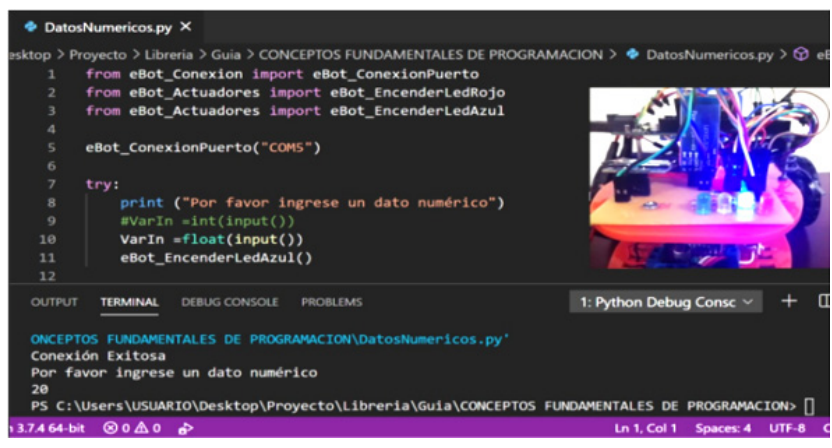

Fig. 10. Ejercicio Elemento de Aprendizaje 1

\section{- Elemento de aprendizaje 2}

Esta actividad hace referencia al tema de estructuras selectivas, que son aquellas que se utilizan cuando en el desarrollo de la solución de un problema se debe tomar una decisión, para establecer un proceso o señalar un camino alternativo a seguir. Dentro de estas estructuras se encuentran:

- Estructuras selectivas simple (Si Entonces)

- Estructuras selectivas doble (Si Entonces/ SiNo)

- Estructuras selectivas anidadas

En la Fig. 11, se muestra una actividad la cual hace referencia a uso de estructuras selectivas anidadas y su resultado en E-BOT, como se evidencia en esta actividad se activaron dos módulos del robot (LEDs, OLED).

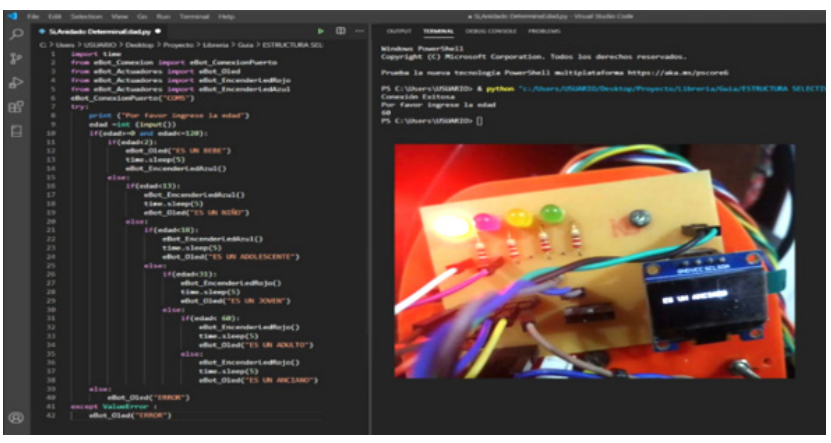

Fig. 11. Ejercicio Elemento de Aprendizaje 2

\section{- Elemento de aprendizaje 3}

Este elemento abordó el tema de las estructuras repetitivas las cuales son sentencias que se utilizan cuando se quiere que un conjunto de instrucciones se ejecuten un cierto número de veces. En la Fig. 12, se muestra un ejemplo que hace uso de la esctructura "FOR", donde se le indica a

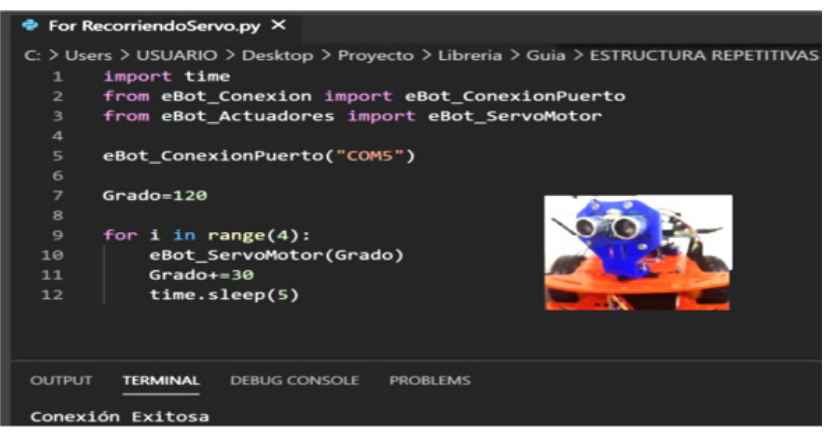

Fig. 12. Ejercicio Elemento de Aprendizaje 3

Rev. Ingeniería, Matemáticas y Ciencias de la Información Vol. 8 / Núm. 16 / julio - diciembre de 2021; 65-76 
E-BOT que debe girar el servomotor $30^{\circ}$ iniciando en $120^{\circ}$ hasta terminar en $180^{\circ}$.

\section{CONCLUSIONES}

Se realizó el diseño y construcción de una plataforma robotica móvil (E-BOT), tanto en su parte mecánica como electrónica. Cabe resaltar que, donde se presentaron mayores inconvenientes fue en la parte mecánica, debido a que se debieron realizar varias adecuaciones y adaptar nuevos elementos que no se tenían contemplados para un correcto funcionamiento del sistema. En cuanto a la parte electrónica no se presentaron mayores contratiempos debido a la utilización de la placa de Arduino ya que su enorme versatilidad permitió controlar de manera sencilla todos los elementos (actuadores y sensores) que integra E-BOT.

Se implementó un software de verificación que permite el control de los actuadores y monitoreo de los sensores en tiempo real, por medio de una variedad de módulos previamente programados y configurados en Python.

Se diseña una serie de elementos de aprendizaje que permitirán la enseñanza dinámica de temas relacionados a programación con E-BOT como un medio de aprendizaje, las cuales tendrán una interacción del software con los actuadores y sensores que se adecuaron en el robot. Esto es posible gracias a la construcción de una serie de librerías que permiten el control de dichos elementos. Es importante indicar que estas actividades son dirigidas a personas que no tienen ningún tipo de conocimiento o conocimiento muy básico en programación.

\section{TRabajos fUtUROS}

Para trabajos futuros sobre este proyecto se plantea realizar un estudio de como E-BOT mendiante sus elementos puede facilitar la enseñanza y ayudar en el desarrollo de competencias de aprendizaje en conceptos relacionados con programación, realizando actividades didácticas basadas en el uso del robot y, después comparando dichos resultados con una metodología más tradicional focalizada en una exposición magistral de los contenidos sin la utilización del robot.
Adicionalmente se podría maximizar el alcance del robot adecuando más sensores o actuadores según la capacidad permitida, dando así paso a funcionalidades nuevas que se puedan integrar con las funciones ya existentes para realizar unos nuevos elementos de aprendizaje o realizar la integracion de varios sensores del robot que permitan la creación de aplicaciones mas complejas.

\section{REFERENCIAS}

[1] R. Casado Fernandez \& M. Checa Romero. Robótica y Proyectos STEAM: Desarrollo de la creatividad en las aulas de Educación Primaria. Píxel-BIT Revista de Medios y Educación - 2020 - N 58. España. 2020.

[2] C. González Ayerbe, Manejadores de sensores y actuadores lego mindstorms para raspberry pi y marte os: (Lego Mindstorms sensors and actuatorsdrivers for Raspberry Pi and marte os). Universidad de Cantabria. España. 2017.

[3] F. Bravo Sánchez \& A. Forero Guzmán, La robótica como un recurso para facilitar el aprendizaje y desarrollo de competencias generales; Teoría de la Educación. Educación y Cultura en la Sociedad de la Información, vol. 13, núm. 2. Colombia. 2012.

[4] C. Angulo. Usos y beneficios de la robótica en las aulas. https://www.upc.edu/latevaupc/usos-ybeneficios-robotica-las-aulas/. 2016.

[5] E. Garnica Estrada, Robots herramientas para las aulas de clase, RIMCI, vol. 1, n. ${ }^{\circ} 1$. http:// ojs.urepublicana.edu.co/index.php/ingenieria/ article/view/219. 2014.

[6] A. Muñoz Ramírez y J. Gómez, Experiencia docente en Automática empleando un robot móvil como elemento motivador de metodologías activas; 5 th International Conference on Educational Innovation in Technical Careers. España. 2017.

[7] L. Londoño, Análisis, diseño e implementación de una plataforma triada integrada por Raspberry $\mathrm{Pi}$, Arduino y dispositivos móviles con conectividad en red local, para la enseñanza de las ciencias naturales: estudio de caso en Física. Universidad Nacional de Colombia Facultad de ciencias. Colombia. 2018.

[8] J. Gustavo, S. Astudillo, P. Willging, D. Segovia, L. Castro y J. Distel, Estrategias innovadoras en los procesos de Enseñanza y de Aprendizajes de Informática. XX Workshop de Investigadores en Ciencias de la Computación. Argentina. 2018.

[9] J. Poco Paredes, La robótica educativa y su influencia en el aprendizaje colaborativo en estudiantes 
de primero de secundaria de la I.E. General José de San Martín; Universidad Nacional de San Agustin. Peru. 2018.

[10] G. Peralta Buitrago, Robótica educativa: (Una estrategia en el desarrollo de la creatividad y las capacidades en educación en tecnología). Instituto Latinoamericano de altos estudios -ILAE. Colombia. 2015.

[11] C. Guimarães, J. L. Rubio Tamayo, R. Ventura, B. Henriques. Robótica para los procesos de enseñanza de la disciplina mecatrónica: Desarrollo del prototipo Edubot-v2. Actas icono14 - No 16 I III Congreso Internacional Sociedad Digital. España. 2014.

[12] J. M. García, Robótica Educativa. La programación como proceso educativo. RED. Revista de Educación a Distancia. Número 46; Uruguay. 2015.

[13] A. Hurtado, N. Santamaría, La robótica en la enseñanza de las ciencias en primaria, una experiencia con Bee-Bot; Creativity and Educational Innovation Review No 3. España. 2019.

[14] R. Cózar, S. González, J. M. Merino, R. Villena, Aprendiendo con robots en edades tempranas; CIED-Centro Interdisciplinar de Estudios Educaciones.España. 2019.

[15] J. S. Duana, L. Defelippe, J. Benítez, C. Leonardi, Plataforma educativa basada en robótica de bajo costo para la enseñanza de la programación; Instituto de Investigación en Tecnología Informática Avanzada (INTIA). Facultad de Ciencias Exactas, Universidad Nacional del Centro de la Provincia de Buenos Aires (UNICEN). Argentina. 2019.
[16] M. Pinto, N. Barrera, W. J. Pérez, Uso de la Robótica Educativa como Herramienta en los procesos de Enseñanza. Universidad Pedagógica y Tecnológica de Colombia Grupo de Investigación en Robótica y Automatización Industrial, GIRA. Colombia. 2015.

[17] P. Aguayo S. Introducción al microcontrolador. https:// www.academia.edu/17264316/Micro. 2014.

[18] O. Artero, Arduino: Curso práctico de formación, Alfaomega. México. 2013.

[19] G. Cujilema, M. A. Véliz, Implementación de un Robot MegaSumo Radiocontrolado y Autónomo utilizando microcontroladores PIC.universidad católica de Santiago de Guayaquil.Ecuador. 2016.

[20] M. Costanzo, M. Boggia, I. Rodríguez, A. De Giusti. Cloud Robotics: Vehículo autónomo conectado a AWS.Consejo Nacional de Investigaciones Científicas y Técnicas (CONICET). Argentina. 2018.

[21] M. Sánchez, Estudio E Implementación De La Tecnología Oled.Escuela Politecnica superior de Gandia. España. 2012.

[22] F. R. Machín, Mejoras en el Control y Sistema de Comunicaciones del Robot Ballbot-Ull. Universidad de la Laguna. España. 2017.

[23] G. López y S. Margni, Construcción de Robots a Bajo Costo Motores y Sensores en Robótica. Universidad de la República Oriental del Uruguay. Uruguay. 2003. 